\title{
History of quaternion and Clifford Fourier transforms and wavelets
}

\author{
Fred Brackx, Eckhard Hitzer and Stephen J. Sangwine
}

\begin{abstract}
We survey the historical development of quaternion and Clifford Fourier transforms and wavelets.
\end{abstract}

Keywords. Quaternions, Clifford Algebra, Fourier Transforms, Wavelet Transforms.

The development of hypercomplex Fourier transforms and wavelets has taken place in several different threads, reflected in the overview of the subject presented in this chapter. We present in Section 1 an overview of the development of quaternion Fourier transforms, then in Section 2 the development of Clifford Fourier transforms. Finally, since wavelets are a more recent development, and the distinction between their quaternion and Clifford algebra approach has been much less pronounced than in the case of Fourier transforms, Section 3 reviews the history of both quaternion and Clifford wavelets.

We recognise that the history we present here may be incomplete, and that work by some authors may have been overlooked, for which we can only offer our humble apologies.

\section{Quaternion Fourier Transforms (QFT)}

\subsection{Major Developments in the History of the Quaternion Fourier Trans- form}

Quaternions [51] were first applied to Fourier transforms by Ernst [49, $\S 6.4 .2]$ and Delsuc [41, Eqn. 20] in the late 1980s, seemingly without knowledge of the earlier work of Sommen [90, 91] on Clifford Fourier and Laplace transforms further explained in Section 2.2. Ernst and Delsuc's quaternion transforms were two-dimensional (that is they had two independent variables) and proposed for application to nuclear magnetic resonance (NMR) imaging. Written in terms of two independent time variables ${ }^{1} t_{1}$ and $t_{2}$, the forward transforms

\footnotetext{
${ }^{1}$ The two independent time variables arise naturally from the formulation of twodimensional NMR spectroscopy.
} 
were of the following form ${ }^{2}$ :

$$
\mathcal{F}\left(\omega_{1}, \omega_{2}\right)=\int_{-\infty}^{\infty} \int_{-\infty}^{\infty} f\left(t_{1}, t_{2}\right) e^{i \omega_{1} t_{1}} e^{j \omega_{2} t_{2}} \mathrm{~d} t_{1} \mathrm{~d} t_{2}
$$

Notice the use of different quaternion basis units $\boldsymbol{i}$ and $\boldsymbol{j}$ in each of the two exponentials, a feature that was essential to maintain the separation between the two dimensions (the prime motivation for using a quaternion Fourier transformation was to avoid the mixing of information that occurred when using a complex Fourier transform - something that now seems obvious, but must have been less so in the 1980s). The signal waveforms/samples measured in NMR are complex, so the quaternion aspect of this transform was essential only for maintaining the separation between the two dimensions. As we will see below, there was some unused potential here.

The fact that exponentials in the above formulation do not commute (with each other, or with the 'signal' function $f$ ), means that other formulations are possible ${ }^{3}$, and indeed Ell in $1992[45,46]$ formulated a transform with the two exponentials positioned either side of the signal function:

$$
\mathcal{F}\left(\omega_{1}, \omega_{2}\right)=\int_{-\infty}^{\infty} \int_{-\infty}^{\infty} e^{i \omega_{1} t_{1}} f\left(t_{1}, t_{2}\right) e^{j \omega_{2} t_{2}} \mathrm{~d} t_{1} \mathrm{~d} t_{2} .
$$

Ell's transform was a theoretical development, but it was soon applied to the practical problem of computing a holistic Fourier transform of a colour image [84] in which the signal samples (discrete image pixels) had three-dimensional values (represented as quaternions with zero scalar parts). This was a major change from the previously intended application in nuclear magnetic resonance, because now the two-dimensional nature of the transform mirrored the two-dimensional nature of the image, and the four-dimensional nature of the algebra used followed naturally from the three-dimensional nature of the image pixels.

Other researchers in signal and image processing have followed Ell's formulation (with trivial changes of basis units in the exponentials) [27, 24, 25], but as with the NMR transforms, the quaternion nature of the transforms was applied essentially to separation of the two independent dimensions of an image (Bülow's work [24, 25] was based on greyscale images, that is with one-dimensional pixel values). Two new ideas emerged in 1998 in a paper by Sangwine and Ell [86]. These were, firstly, the choice of a general root $\mu$ of -1 (a unit quaternion with zero scalar part) rather than a basis unit $(\boldsymbol{i}, \boldsymbol{j}$ or $\boldsymbol{k}$ ) of the quaternion algebra, and secondly, the choice of a single exponential

\footnotetext{
${ }^{2}$ Note, that Georgiev et al use this form of the quaternion Fourier transform (QFT) in Chapter ?? to extend the Bochner-Minlos theorem to quaternion analysis. Moreover, the same form of QFT is extended by Georgiev and Morais in Chapter ?? to a quaternion Fourier-Stieltjes transform.

${ }^{3}$ See Chapter ?? by Ell in this volume with a systematic review of possible forms of quaternion Fourier transformations.
} 
rather than two (giving a choice of ordering relative to the quaternionic signal function):

$$
\mathcal{F}\left(\omega_{1}, \omega_{2}\right)=\int_{-\infty}^{\infty} \int_{-\infty}^{\infty} e^{\mu\left(\omega_{1} t_{1}+\omega_{2} t_{2}\right)} f\left(t_{1}, t_{2}\right) \mathrm{d} t_{1} \mathrm{~d} t_{2} .
$$

This made possible a quaternion Fourier transform of a one-dimensional signal:

$$
\mathcal{F}(\omega)=\int_{-\infty}^{\infty} e^{\mu \omega t} f(t) \mathrm{d} t
$$

Such a transform makes sense only if the signal function has quaternion values, suggesting applications where the signal has three or four independent components. (An example is vibrations in a solid, such as rock, detected by a sensor with three mutually orthogonal transducers, such as a vector geophone.)

Very little has appeared in print about the interpretation of the Fourier coefficients resulting from a quaternion Fourier transform. One interpretation are components of different symmetry, as explained by Ell in Chapter ??. Sangwine and Ell in 2007 published a paper about quaternion Fourier transforms applied to colour images, with a detailed explanation of the Fourier coefficients in terms of elliptical paths in colour space (the $n$-dimensional space of the values of the image pixels in a colour image) [48].

\subsection{Splitting Quaternions and the QFT}

Following the earlier works of Ernst, Ell, Sangwine (see Section 1.1), and Bülow [24, 25], Hitzer thoroughly studied the quaternion Fourier transform (QFT) applied to quaternion-valued functions in [54]. As part of this work a quaternion split

$$
q_{ \pm}=\frac{1}{2}(q \pm \boldsymbol{i} q \boldsymbol{j}), \quad q \in \mathbb{H},
$$

was devised and applied, which led to a better understanding of $G L\left(\mathbb{R}^{2}\right)$ transformation properties of the QFT spectrum of two-dimensional images, including colour images, and opened the way to a generalization of the QFT concept to a full spacetime Fourier transformation (SFT) for spacetime algebra $C \ell_{3,1}$-valued signals.

This was followed up by the establishment of a fully directional (opposed to componentwise) uncertainty principle for the QFT and the SFT [58]. Independently Mawardi et al [77] established a componentwise uncertainty principle for the QFT.

The QFT with a Gabor window was treated by Bülow [24], a study which has been continued by Mawardi et al in [1].

Hitzer reports in [59] initial results (obtained in co-operation with Sangwine) about a further generalization of the QFT to a general form of orthogonal 2D planes split (OPS-) QFT, where the split (1.5) with respect to two orthogonal pure quaternion units $\boldsymbol{i}, \boldsymbol{j}$ is generalized to a steerable split with 
respect to any two pure unit quaternions $f, g \in \mathbb{H}, f^{2}=g^{2}=-1$. This approach is fully elaborated upon in a contribution to the current volume (see Chapter ??). Note that the Cayley-Dickson form [87] of quaternions and the related simplex/perplex split [47] are obtained for $f=g=\boldsymbol{i}$ (or more general $f=g=\mu$ ), which is employed in Chapter ?? for a novel spectral analysis of non-stationary improper complex signals.

\section{Clifford Fourier Transformations in Clifford's Geometric Algebra}

W.K. Clifford introduced (Clifford) geometric algebras in 1876 [28]. An introduction to the vector and multivector calculus, with functions taking values in Clifford algebras, used in the field of Clifford Fourier transforms (CFT) can be found in $[53,52]$. A tutorial introduction to CFTs and Clifford wavelet transforms can be found in [55]. The Clifford algebra application survey [65] contains an up to date section on applications of Clifford algebra intergral transforms, including CFTs, QFTs and wavelet transforms ${ }^{4}$.

\subsection{How Clifford Algebra Square Roots of -1 Lead to Clifford Fourier Transformations}

In 1990 Jancewicz defined a trivector Fourier transformation

$$
\mathcal{F}_{3}\{g\}(\boldsymbol{\omega})=\int_{\mathbb{R}^{3}} g(\mathbf{x}) e^{-i_{3} \mathbf{x} \cdot \boldsymbol{\omega}} d^{3} \mathbf{x}, \quad i_{3}=\boldsymbol{e}_{1} \boldsymbol{e}_{2} \boldsymbol{e}_{3}, \quad g: \mathbb{R}^{3} \rightarrow C \ell_{3,0},
$$

for the electromagnetic field ${ }^{5}$ replacing the imaginary unit $i \in \mathbb{C}$ by the trivector $i_{3}, i_{3}^{2}=-1$, of the geometric algebra $C \ell_{3,0}$ of three-dimensional Euclidean space $\mathbb{R}^{3}=\mathbb{R}^{3,0}$ with ortho-normal vector basis $\left\{\boldsymbol{e}_{1}, \boldsymbol{e}_{2}, \boldsymbol{e}_{3}\right\}$.

In [50] Felsberg makes use of signal embeddings in low dimensional Clifford algebras $\mathbb{R}_{2,0}$ and $\mathbb{R}_{3,0}$ to define his Clifford-Fourier transform (CFT) for one-dimensional signals as

$$
\mathcal{F}_{1}^{f e}[f](\underline{u})=\int_{\mathbb{R}} \exp \left(-2 \pi i_{2} \underline{u} \underline{x}\right) f(\underline{x}) d \underline{x}, \quad i_{2}=\boldsymbol{e}_{1} \boldsymbol{e}_{2}, \quad f: \mathbb{R} \rightarrow \mathbb{R},
$$

where he uses the pseudoscalar $i_{2} \in C \ell_{2,0}, i_{2}^{2}=-1$. For two-dimensional signals $^{6}$ he defines the CFT as

$$
\mathcal{F}_{2}^{f e}[f](\underline{u})=\int_{\mathbb{R}^{2}} \exp \left(-2 \pi i_{3}<\underline{u}, \underline{x}>\right) f(\underline{x}) d \underline{x}, \quad f: \mathbb{R}^{2} \rightarrow \mathbb{R}^{2},
$$

\footnotetext{
${ }^{4}$ Fourier and wavelet transforms provide alternative signal and image representations. See Chapter ?? for a spinorial representation and Chapter ?? by Li and Qian for a sparse representation of signals in a Hardy space dictionary (of elementary wave forms) over a unit disk.

${ }^{5}$ Note also Chapter ?? in this volume, in which Bernstein considers optical coherence tomography, formulating the Maxwell equations with the Dirac operator and Clifford algebra. ${ }^{6}$ Note in this context the spinor representation of images by Batard and Berthier in Chapter ?? of this volume. The authors apply a CFT in $C \ell_{3,0}$ to the spinor represenation, which uses in the exponential kernel an adapted choice of bivector, that belongs to the orthonormal frame of the tangent bundle of an oriented two-dimensional Riemannian manifold, isometrically immersed in $\mathbb{R}^{3}$.
} 
where he uses the pseudoscalar $i_{3} \in C \ell_{3,0}$. It is used a.o. to introduce a concept of two-dimensional analytic signal. Together with Bülow and Sommer, Felsberg applied these CFTs to image stucture processing (key-notion: structure multivector) $[50,24]$.

Ebling and Scheuermann [44, 43] consequently applied to vector signal processing in two- and three dimensions, respectively, the following twodimensional CFT

$$
\mathcal{F}_{2}\{f\}(\boldsymbol{\omega})=\int_{\mathbb{R}^{2}} f(\mathbf{x}) e^{-i_{2} \mathbf{X} \cdot \boldsymbol{\omega}} d^{2} \mathbf{x}, \quad f: \mathbb{R}^{2} \rightarrow \mathbb{R}^{2},
$$

with Clifford Fourier kernel

$$
\exp \left(-\boldsymbol{e}_{1} \boldsymbol{e}_{2}\left(\omega_{1} x_{1}+\omega_{2} x_{2}\right)\right)
$$

and the three-dimensional CFT (2.1) of Jancewicz with Clifford Fourier kernel

$$
\exp \left(-\boldsymbol{e}_{1} \boldsymbol{e}_{2} \boldsymbol{e}_{3}\left(\omega_{1} x_{1}+\omega_{2} x_{2}+\omega_{3} x_{3}\right)\right) \text {. }
$$

An important integral operation defined and applied in this context by Ebling and Scheuermann was the Clifford convolution. These Clifford-Fourier transforms and the corresponding convolution theorems allow Ebling and Scheuermann for a.o. the analysis of vector-valued patterns in the frequency domain.

Note that the latter Fourier kernel (2.6) has also been used by Mawardi and Hitzer in $[78,63,78]$ to define their Clifford-Fourier transform of threedimensional multivector signals: that means, they researched the properties of $\mathcal{F}_{3}\{g\}(\boldsymbol{\omega})$ of $(2.1)$ in detail when applied to full multivector signals $g$ : $\mathbb{R}^{3} \rightarrow C \ell_{3,0}$. This included an investigation of the uncertainty inequality for this type of CFT. They subsequently generalized $\mathcal{F}_{3}\{g\}(\boldsymbol{\omega})$ to dimensions $n=3(\bmod 4)$, i.e. $n=3,7,11, \ldots$,

$$
\mathcal{F}_{n}\{g\}(\boldsymbol{\omega})=\int_{\mathbb{R}^{n}} g(\mathbf{x}) e^{-i_{n} \mathbf{x} \cdot \boldsymbol{\omega}^{n}} d^{n}, \quad g: \mathbb{R}^{n} \rightarrow C \ell_{n, 0},
$$

which is straight-forward, since for these dimensions the pseudoscalar $i_{n}=$ $\boldsymbol{e}_{1} \ldots \boldsymbol{e}_{n}$ squares to -1 and is central [64], i.e., it commutes with every other multivector belonging to $C \ell_{n, 0}$. A little less trivial is the generalization of $\mathcal{F}_{2}\{f\}(\boldsymbol{\omega})$ of $(2.4)$ to

$$
\mathcal{F}_{n}\{f\}(\boldsymbol{\omega})=\int_{\mathbb{R}^{n}} f(\mathbf{x}) e^{-i_{n} \mathbf{x} \cdot \boldsymbol{\omega}} d^{n} \mathbf{x}, \quad f: \mathbb{R}^{n} \rightarrow C \ell_{n, 0},
$$

with $n=2(\bmod 4)$, i.e. $n=2,6,10 \ldots$, because in these dimensions the pseudoscalar $i_{n}=\boldsymbol{e}_{1} \ldots \boldsymbol{e}_{n}$ squares to -1 , but it ceases to be central. So the relative order of the factors in $\mathcal{F}_{n}\{f\}(\boldsymbol{\omega})$ becomes important, see [66] for a systematic investigation and comparison.

In the context of generalizing quaternion Fourier transforms (QFT) via algebra isomorphisms to higher dimensional Clifford algebras, Hitzer [54] constructed a spacetime Fourier transform (SFT) in the full algebra of spacetime $C \ell_{3,1}$, which includes the CFT (2.1) as a partial transform of space. Implemented analogous (isomorphic) to the orthogonal 2D planes split of quaternions, the SFT permits a natural spacetime split, which algebraically splits the SFT into right- and left propagating multivector wave packets. This analysis 
allows to compute the effect of Lorentz transformations on the spectra of these wavepackets, as well as a 4D directional spacetime uncertainty formula [58] for spacetime signals.

Mawardi et al extended the CFT $\mathcal{F}_{2}\{f\}(\boldsymbol{\omega})$ of (2.4) to a windowed CFT in [76]. Fu et al establish in Chapter ?? a strong version of Heisenberg's uncertainty principle for Gabor-windowed CFTs.

In Chapter ?? in this volume, Bujack, Scheuermann, and Hitzer, expand the notion of Clifford Fourier transform to include multiple left and right exponential kernel factors, in which commuting (or anticommuting) blades, that square to -1 , replace the complex unit $i \in \mathbb{C}$, thus managing to include most practically used CFTs in a single comprehensive framework. Based on this they have also constructed a general CFT convolution theorem [23].

Spurred by the systematic investigation of (complex quaternion) biquaternion square roots of -1 in $C \ell_{3,0}$ by Sangwine [85], Hitzer and Ablamowicz [62] systematically investigated the explicit equations and solutions for square roots of -1 in all real Clifford algebras $C \ell_{p, q}, p+q \leq 4$. This investigation is continued in the present volume in Chapter ?? by Hitzer, Helmstetter and Ablamowicz for all square roots of -1 in all real Clifford algberas $C \ell_{p, q}$ without restricting the value of $n=p+q$. One important motivation for this is the relevance of the Clifford algebra square roots of -1 for the general construction of CFTs, where the imaginary unit $i \in \mathbb{C}$ is replaced by a $\sqrt{-1} \in C \ell_{p, q}$, without restriction to pseudoscalars or blades.

Based on the knowlege of square roots of -1 in real Clifford algebras $C \ell_{p, q},[60]$ develops a general $\mathrm{CFT}$ in $C \ell_{p, q}$, wherein the complex unit $i \in \mathbb{C}$ is replaced by any square root of -1 chosen from any component and (or) conjugation class of the submanifold of square roots of -1 in $C \ell_{p, q}$, and details its properties, including a convolution theorem. A similar general approach is taken in [61] for the construction of two-sided CFTs in real Clifford algebras $C \ell_{p, q}$, freely choosing two square roots from any one or two components and (or) conjugation classes of the submanifold of square roots of -1 in $C \ell_{p, q}$. These transformations are therefore generically steerable.

This algebraically motivated approach may in the future be favorably combined with group theoretic, operator theoretic and spinorial approaches, to be discussed in the following.

\subsection{The Clifford Fourier Transform in the Light of Clifford Analysis}

Two robust tools used in image processing and computer vision for the analysis of scalar fields are convolution and Fourier transformation. Several attempts have been made to extend these methods to two- and threedimensional vector fields and even multi-vector fields. Let us give an overview of those generalized Fourier transforms.

In [25] Bülow and Sommer define a so-called quaternionic Fourier transform of two-dimensional signals $f\left(x_{1}, x_{2}\right)$ taking their values in the algebra $\mathbb{H}$ of real quaternions. Note that the quaternion algebra $\mathbb{H}$ is nothing else but (isomorphic to) the Clifford algebra $C \ell_{0,2}$ where, traditionally, the basis vectors are denoted by $i$ and $j$, with $i^{2}=j^{2}=-1$, and the bivector by 
$\boldsymbol{k}=\boldsymbol{i} \boldsymbol{j}$. In terms of these basis vectors this quaternionic Fourier transform takes the form

$$
\mathcal{F}^{q}[f]\left(u_{1}, u_{2}\right)=\int_{\mathbb{R}^{2}} \exp \left(-2 \pi \boldsymbol{i} u_{1} x_{1}\right) f\left(x_{1}, x_{2}\right) \exp \left(-2 \pi \boldsymbol{j} u_{2} x_{2}\right) d \underline{x}
$$

Due to the non-commutativity of the multiplication in $\mathbb{H}$, the convolution theorem for this quaternionic Fourier transform is rather complicated, see also [23].

This is also the case for its higher dimensional analogue, the so-called Clifford-Fourier transform ${ }^{7}$ in $C \ell_{0, m}$ given by

$$
\mathcal{F}^{c l}[f](\underline{u})=\int_{\mathbb{R}^{m}} f(\underline{x}) \exp \left(-2 \pi \boldsymbol{e}_{1} u_{1} x_{1}\right) \ldots \exp \left(-2 \pi \boldsymbol{e}_{m} u_{m} x_{m}\right) d \underline{x}
$$

Note that for $m=1$ and interpreting the Clifford basis vector $\boldsymbol{e}_{1}$ as the imaginary unit $i$, the Clifford-Fourier transform (2.10) reduces to the standard Fourier transform on the real line, while for $m=2$ the quaternionic Fourier transform (2.9) is recovered when restricting to real signals.

Finally Bülow and Sommer also introduce a so-called commutative hypercomplex Fourier transform given by

$$
\mathcal{F}^{h}[f](\underline{u})=\int_{\mathbb{R}^{m}} f(\underline{x}) \exp \left(-2 \pi \sum_{j=1}^{m} \tilde{e}_{j} u_{j} x_{j}\right) d \underline{x}
$$

where the basis vectors $\left(\tilde{\boldsymbol{e}}_{1}, \ldots, \tilde{\boldsymbol{e}}_{m}\right)$ obey the commutative multiplication rules $\tilde{\boldsymbol{e}}_{j} \tilde{\boldsymbol{e}}_{k}=\tilde{\boldsymbol{e}}_{k} \tilde{\boldsymbol{e}}_{j}, j, k=1, \ldots, m$, while still retaining $\tilde{\boldsymbol{e}}_{j}^{2}=-1, j=1, \ldots, m$. This commutative hypercomplex Fourier transform offers the advantage of a simple convolution theorem.

The hypercomplex Fourier transforms $\mathcal{F}^{q}, \mathcal{F}^{c l}$ and $\mathcal{F}^{h}$ enable Bülow and Sommer to establish a theory of multi-dimensional signal analysis and in particular to introduce the notions of multi-dimensional analytic signal ${ }^{8}$, Gabor filter, instantaneous and local amplitude and phase, etc.

In this context the Clifford Fourier transformations by Felsberg [50] for one- and two-dimensional signals, by Ebling and Scheuermann for twoand three-dimensional vector signal processing $[44,43]$, and by Mawardi and Hitzer for general multivector signals in $C \ell_{3,0}[78,63,78]$, and their respective kernels, as already reviewed in Section 2.1, should also be considered.

The above mentioned Clifford-Fourier kernel of Bülow and Sommer

$$
\exp \left(-2 \pi \boldsymbol{e}_{1} u_{1} x_{1}\right) \cdots \exp \left(-2 \pi \boldsymbol{e}_{m} u_{m} x_{m}\right)
$$

was in fact already introduced in [19] and [89] as a theoretical concept in the framework of Clifford analysis. This generalized Fourier transform was further elaborated by Sommen in $[90,91]$ in connection with similar generalizations of the Cauchy, Hilbert and Laplace transforms. In this context also the work of Li, McIntosh and Qian should be mentioned; in [72] they generalize the standard multi-dimensional Fourier transform of a function in $\mathbb{R}^{m}$, by

\footnotetext{
${ }^{7}$ Note that in this volume Mawardi establishes in Chapter ?? a windowed version of the CFT (2.10).

${ }^{8}$ See also Chapter ?? by Girard et al and Chapter ?? by Bernstein et al in this volume.
} 
extending the Fourier kernel $\exp (i\langle\underline{\xi}, \underline{x}\rangle)$ to a function which is holomorphic in $\mathbb{C}^{m}$ and monogenic ${ }^{9}$ in $\mathbb{R}^{m+1}$.

In $[15,16,18]$ Brackx, De Schepper and Sommen follow another philosophy in their construction of a Clifford-Fourier transform. One of the most fundamental features of Clifford analysis is the factorization of the Laplace operator. Indeed, whereas in general the square root of the Laplace operator is only a pseudo-differential operator, by embedding Euclidean space into a Clifford algebra, one can realize $\sqrt{-\Delta_{m}}$ as the Dirac operator $\partial_{\underline{x}}$. In this way Clifford analysis spontaneously refines harmonic analysis. In the same order of ideas, Brackx et al decided to not replace nor to improve the classical Fourier transform by a Clifford analysis alternative, since a refinement of it automatically appears within the language of Clifford analysis. The key step to making this refinement apparent is to interpret the standard Fourier transform as an operator exponential:

$$
\mathcal{F}=\exp \left(-i \frac{\pi}{2} \mathcal{H}\right)=\sum_{k=0}^{\infty} \frac{1}{k !}\left(-i \frac{\pi}{2}\right)^{k} \mathcal{H}^{k}
$$

where $\mathcal{H}$ is the scalar operator

$$
\mathcal{H}=\frac{1}{2}\left(-\Delta_{m}+r^{2}-m\right) .
$$

This expression links the Fourier transform with the Lie algebra $\mathfrak{s l}_{2}$ generated by $\Delta_{m}$ and $r^{2}=|x|^{2}$ and with the theory of the quantum harmonic oscillator determined by the Hamiltonian $-\frac{1}{2}\left(\Delta_{m}-r^{2}\right)$. Splitting the operator $\mathcal{H}$ into a sum of Clifford algebra-valued second order operators containing the angular Dirac operator $\Gamma$, one is led, in a natural way, to a pair of transforms $\mathcal{F}_{\mathcal{H}^{ \pm}}$, the harmonic average of which is precisely the standard Fourier transform:

$$
\mathcal{F}_{\mathcal{H}^{ \pm}}=\exp \left(\frac{i \pi m}{4}\right) \exp \left(\mp \frac{i \pi \Gamma}{2}\right) \exp \left(\frac{i \pi}{4}\left(\Delta_{m}-r^{2}\right)\right) .
$$

For the special case of dimension two, Brackx et al obtain a closed form for the kernel of the integral representation of this Clifford-Fourier transform leading to its internal representation

$$
\mathcal{F}_{\mathcal{H}^{ \pm}}[f](\underline{\xi})=\mathcal{F}_{\mathcal{H}^{ \pm}}[f]\left(\xi_{1}, \xi_{2}\right)=\frac{1}{2 \pi} \int_{\mathbb{R}^{2}} \exp \left( \pm \boldsymbol{e}_{12}\left(\xi_{1} x_{2}-\xi_{2} x_{1}\right)\right) f(\underline{x}) d \underline{x},
$$

which enables the generalization of the calculation rules for the standard Fourier transform both in the $L_{1}$ and in the $L_{2}$ context. Moreover, the Clifford-Fourier transform of Ebling and Scheuermann

$$
\mathcal{F}^{e}[f](\underline{\xi})=\int_{\mathbb{R}^{2}} \exp \left(-e_{12}\left(x_{1} \xi_{1}+x_{2} \xi_{2}\right)\right) f(\underline{x}) d \underline{x},
$$

\footnotetext{
${ }^{9}$ See also in this volume chapter ?? by Moya-Sánchez and Bayro-Corrochano on the application of atomic function based monogenic signals.
} 
can be expressed in terms of the Clifford-Fourier transform:

$$
\mathcal{F}^{e}[f](\underline{\xi})=2 \pi \mathcal{F}_{\mathcal{H}^{ \pm}}[f]\left(\mp \xi_{2}, \pm \xi_{1}\right)=2 \pi \mathcal{F}_{\mathcal{H}^{ \pm}}[f]\left( \pm \boldsymbol{e}_{12} \underline{\xi}\right),
$$

taking into account that, under the isomorphism between the Clifford algebras $C \ell_{2,0}$ and $C \ell_{0,2}$, both pseudoscalars are isomorphic images of each other.

The question whether $\mathcal{F}_{\mathcal{H}^{ \pm}}$can be written as an integral transform is answered positively in the case of even dimension by De Bie and $\mathrm{Xu}$ in [39]. The integral kernel of this transform is not easy to obtain and looks quite complicated. In the case of odd dimension the problem is still open.

Recently, in [35], De Bie and De Schepper have studied the fractional Clifford-Fourier transform as a generalization of both the standard fractional Fourier transform and the Clifford-Fourier transform. It is given as an operator exponential by

$$
\mathcal{F}_{\alpha, \beta}=\exp \left(\frac{i \alpha m}{2}\right) \exp (i \beta \Gamma) \exp \left(\frac{i \alpha}{2}\left(\Delta_{m}-r^{2}\right)\right)
$$

For the corresponding integral kernel a series expansion is obtained, and, in the case of dimension two, an explicit expression in terms of Bessel functions.

The above, more or less chronological, overview of generalized Fourier transforms in the framework of quaternionic and Clifford analysis, gives the impression of a medley of ad hoc constructions. However there is a structure behind some of these generalizations, which becomes apparent when, as already slightly touched upon above, the Fourier transform is linked to group representation theory, in particular the Lie algebras $\mathfrak{s l}_{2}$ and $\mathfrak{o s p}(1 \mid 2)$. This unifying character is beautifully demonstrated by De Bie in the overview paper [34], where, next to an extensive bibliography, also new results on some of the transformations mentioned below can be found. It is shown that using realizations of the Lie algebra $\mathfrak{s l}_{2}$ one is lead to scalar generalizations of the Fourier transform, such as:

(i) the fractional Fourier transform, which is, as the standard Fourier transform, invariant under the orthogonal group; this transform has been reinvented several times as well in mathematics as in physics, and is attributed to Namias [81], Condon [30], Bargmann [2], Collins [29], Moshinsky and Quesne [80]; for a detailed overview of the theory and recent applications of the fractional Fourier transform we refer the reader to $[82]$;

(ii) the Dunkl transform, see e.g. [42], where the symmetry is reduced to that of a finite reflection group;

(iii) the radially deformed Fourier transform, see e.g. [71], which encompasses both the fractional Fourier and the Dunkl transform;

(iv) the super Fourier transform, see e.g. [33, 31], which is defined in the context of superspaces and is invariant under the product of the orthogonal with the symplectic group.

Realizations of the Lie algebra $\mathfrak{o s p}(1 \mid 2)$, on the contrary, need the framework of Clifford analysis, and lead to: 
(v) the Clifford-Fourier transform and the fractional Clifford-Fourier transform, both already mentioned above; meanwhile an entire class of CliffordFourier transforms has been thoroughly studied in [36];

(vi) the radially deformed hypercomplex Fourier transform, which appears as a special case in the theory of radial deformations of the Lie algebra $\mathfrak{o s p}(1 \mid 2)$, see [38, 37], and is a topic of current research, see [32].

\section{Quaternion and Clifford Wavelets}

\subsection{Clifford Wavelets in Clifford Analysis}

The interest of the Ghent Clifford Research Group for generalizations of the Fourier transform in the framework of Clifford analysis, grew out from the study of the multidimensional Continuous Wavelet Transform in this particular setting. Clifford-wavelet theory, however restricted to the continuous wavelet transform, was initiated by Brackx and Sommen in [20] and further developed by N. De Schepper in her PhD thesis [40]. The Clifford-wavelets originate from a mother wavelet not only by translation and dilation, but also by rotation, making the Clifford-wavelets appropriate for detecting directional phenomena. Rotations are implemented as specific actions on the variable by a spin element, since, indeed, the special orthogonal group $\mathrm{SO}(m)$ is doubly covered by the spin group $\operatorname{Spin}(m)$ of the real Clifford algebra $C \ell_{0, m}$. The mother wavelets themselves are derived from intentionally devised orthogonal polynomials in Euclidean space. It should be noted that these orthogonal polynomials are not tensor products of one-dimensional ones, but genuine multidimensional ones satisfying the usual properties such as a Rodrigues formula, recurrence relations, and differential equations. In this way multidimensional Clifford wavelets were constructed grafted on the Hermite polynomials [21], Laguerre polynomials [14], Gegenbauer polynomials [13], Jacobi polynomials [17], and Bessel functions [22].

Taking the dimension $m$ to be even, say $m=2 n$, introducing a complex structure, i. e. an $\mathrm{SO}(2 n)$-element squaring up to -1 , and considering functions with values in the complex Clifford algebra $\mathbb{C}_{2 n}$, so-called Hermitian Clifford analysis originates as a refinement of standard or Euclidean Clifford analysis. It should be noticed that the traditional holomorphic functions of several complex variables appear as a special case of Hermitian Clifford analysis, when the function values are restricted to a specific homogeneous part of spinor space. In this Hermitian setting the standard Dirac operator, which is invariant under the orthogonal group $\mathrm{O}(m)$, is split into two Hermitian Dirac operators, which are now invariant under the unitary group $\mathrm{U}(n)$. Also in this Hermitian Clifford analysis framework, multidimensional wavelets have been introduced by Brackx, H. De Schepper and Sommen [11, 12], as kernels for a Hermitian Continuous Wavelet Transform, and (generalized) Hermitian Clifford-Hermite polynomials have been devised to generate the corresponding Hermitian wavelets [9, 10]. 


\subsection{Further Developments in Quaternion and Clifford Wavelet Theory}

Clifford algebra multiresolution analysis (MRA) has been pioneered by M. Mitrea [79]. Important are also the electromagnetic signal application oriented developments of Clifford algebra wavelets by G. Kaiser [70, 67, 68, 69].

Quaternion MRA Wavelets with applications to image analysis have been developed in [92] by Traversoni. Clifford algebra multiresolution analysis has been applied by Bayro-Corrochano $[5,3,4]$ to: Clifford wavelet neural networks (information processing), also considering quaternionic MRA, a quaternionic wavelet phase concept, as well as applications to (e.g. robotic) motion estimation and image processing.

Beyond this Zhao and Peng [94] established a theory of quaternionvalued admissible wavelets. Zhao [93] studied Clifford algebra-valued admissible (continuous) wavelets using the complex Fourier transform for the spectral representation. Mawardi and Hitzer [74, 75] extended this to continuos Clifford and Clifford-Gabor wavelets in $C \ell_{3,0}$ using the CFT of (2.1) for the spectral representation. They also studied a corresponding Clifford wavelet transform uncertainty principle. Hitzer $[56,57]$ generalized this approach to continous admissible Clifford algebra wavelets in real Clifford algebras $C \ell_{n, 0}$ of dimensions $n=2,3(\bmod 4)$, i.e. $n=2,3,6,7,10,11, \ldots$. Restricted to $C \ell_{n, 0}$ of dimensions $n=2(\bmod 4)$ this approach has also been taken up in [73].

Kähler et al [26] treated monogenic (Clifford) wavelets over the unit ball. Bernstein studied Clifford continuous wavelet transforms in $L_{0,2}$ and $L_{0,3}[6]$, as well as monogenic kernels and wavelets on the three-dimensional sphere [7]. Bernstein et al [8] further studied Clifford diffusion wavelets on conformally flat cylinders and tori. In the current volume Soulard and Carré extend in Chapter ?? the theory and application of monogenic wavelets to colour image denoising.

\section{References}

[1] M. Bahri, E. Hitzer, R. Ashino, and R. Vaillancourt. Windowed Fourier transform of two-dimensional quaternionic signals. Applied Mathematics and Computation, 216(8):2366-2379, June 2010.

[2] V. Bargmann. On a Hilbert space of analytic functions and an associated integral transform. Communications on Pure and Applied Mathematics, 14(3):187214, Aug. 1961.

[3] E. Bayro-Corrochano. Multi-resolution image analysis using the quaternion wavelet transform. Numerical Algorithms, 39(1-3):35-55, 2005.

[4] E. Bayro-Corrochano. The theory and use of the quaternion wavelet transform. Journal of Mathematical Imaging and Vision, 24:19-35, 2006.

[5] E. Bayro-Corrochano and M. A. de la Torre Gomora. Image processing using the quaternion wavelet transform. In Proceedings of the Iberoamerican Congress on Pattern Recognition, CIARP'2004, pages 612-620, Puebla, Mexico, October 2004 . 
[6] S. Bernstein. Clifford continuous wavelet transforms in $l_{0,2}$ and $l_{0,3}$. In T. E. Simos, G. Psihoyios, and C. Tsitouras, editors, NUMERICAL ANALYSIS AND APPLIED MATHEMATICS: International Conference on Numerical Analysis and Applied Mathematics, volume 1048 of AIP Conference Proceedings, pages 634-637, Psalidi, Kos, Greece, 16-20 September 2008.

[7] S. Bernstein. Spherical singular integrals, monogenic kernels and wavelets on the 3D sphere. Advances in Applied Clifford Algebras, 19(2):173-189, 2009.

[8] S. Bernstein, S. Ebert, and R. S. Krausshar. Diffusion wavelets on conformally flat cylinders and tori. In Simos et al. [88], pages 773-776.

[9] F. Brackx, H. De Schepper, N. De Schepper, and F. Sommen. The generalized Hermitean Clifford-Hermite continuous wavelet transform. In T. E. Simos, G. Psihoyios, and C. Tsitouras, editors, NUMERICAL ANALYSIS AND APPLIED MATHEMATICS: International Conference of Numerical Analysis and Applied Mathematics, volume 936 of AIP Conference Proceedings, pages 721725, Corfu, Greece, 16-20 September 2007.

[10] F. Brackx, H. De Schepper, N. De Schepper, and F. Sommen. Generalized Hermitean Clifford-Hermite polynomials and the associated wavelet transform. Mathematical Methods in the Applied Sciences, 32(5):606-630, 2009.

[11] F. Brackx, H. De Schepper, and F. Sommen. A Hermitian setting for wavelet analysis: the basics. In Proceedings of the 4 th International Conference on Wavelet Analysis and Its Applications, 2005.

[12] F. Brackx, H. De Schepper, and F. Sommen. A theoretical framework for wavelet analysis in a Hermitean Clifford setting. Communications on Pure and Applied Analysis, 6(3):549-567, 2007.

[13] F. Brackx, N. De Schepper, and F. Sommen. The Clifford-Gegenbauer polynomials and the associated continuous wavelet transform. Integral Transforms and Special Functions, 15(5):387-404, 2004.

[14] F. Brackx, N. De Schepper, and F. Sommen. The Clifford-Laguerre continuous wavelet transform. Bulletin of the Belgian Mathematical Society - Simon Stevin, 11(2):201-215, 2004.

[15] F. Brackx, N. De Schepper, and F. Sommen. The Clifford-Fourier transform. Journal of Fourier Analysis and Applications, 11(6):669-681, 2005.

[16] F. Brackx, N. De Schepper, and F. Sommen. The two-dimensional CliffordFourier transform. Journal of Mathematical Imaging and Vision, 26(1):5-18, 2006.

[17] F. Brackx, N. De Schepper, and F. Sommen. Clifford-Jacobi polynomials and the associated continuous wavelet transform in Euclidean space. In Qian et al. [83], pages 185-198.

[18] F. Brackx, N. De Schepper, and F. Sommen. The Fourier transform in Clifford analysis. Advances in Imaging and Electron Physics, 156:55-201, 2009.

[19] F. Brackx, R. Delanghe, and F. Sommen. Clifford Analysis, volume 76. Pitman, Boston, 1982.

[20] F. Brackx and F. Sommen. The continuous wavelet transform in Clifford analysis. In F. Brackx, J. S. R. Chisholm, and V. Souček, editors, Clifford Analysis and its Applications, pages 9-26. Kluwer, 2001. 
[21] F. Brackx and F. Sommen. The generalized Clifford-Hermite continuous wavelet transform. Advances in Applied Clifford Algebras, 11(S1):219-231, Feb. 2001.

[22] F. Brackx and F. Sommen. Clifford-Bessel wavelets in Euclidean space. Mathematical Methods in the Applied Sciences, 25(16-18):1479-1491, Nov./Dec. 2002 .

[23] R. Bujack, G. Scheuermann, and E. Hitzer. A general geometric Fourier transform convolution theorem. Advances in Applied Clifford Algebras, 2012.

[24] T. Bülow. Hypercomplex Spectral Signal Representations for the Processing and Analysis of Images. PhD thesis, University of Kiel, Germany, Institut für Informatik und Praktische Mathematik, Aug. 1999.

[25] T. Bülow and G. Sommer. Hypercomplex signals - a novel extension of the analytic signal to the multidimensional case. IEEE Transactions on Signal Processing, 49(11):2844-2852, Nov. 2001.

[26] P. Cerejeiras, M. Ferreira, and U. Kähler. Monogenic wavelets over unit ball. Zeitschrift für Analysis und ihre Anwendungen, 24(4):841-852, 2005.

[27] V. M. Chernov. Discrete orthogonal transforms with data representation in composition algebras. In Proceedings Scandinavian Conference on Image Analysis, pages 357-364, Uppsala, Sweden, 1995.

[28] W. K. Clifford. On the classification of geometric algebras. In R. Tucker, editor, Mathematical Papers (1882), pages 397-401. Macmillian, London, 1876. Found amongst Clifford's notes, of which the forewords are the abstract that he communicated to the London Mathematical Society on 10th March 1876 (See A. Diek, R. Kantowski, www.nhn.ou.edu/ ski/papers/Clifford/history.ps).

[29] S. A. Collins. Lens-system diffraction integral written in terms of matrix optics. Journal of the Optical Society of America, 60:1168-1177, 1970.

[30] E. U. Condon. Immersion of the Fourier transform in a continuous group of functional transformations. Proceedings of the National Academy of Sciences USA, 23:158-164, 1937.

[31] K. Coulembier and H. De Bie. Hilbert space for quantum mechanics on superspace. Journal of Mathematical Physics, 52, 2011. 063504, 30 pages.

[32] H. De Bie. The kernel of the radially deformed Fourier transform. In preparation.

[33] H. De Bie. Fourier transform and related integral transforms in superspace. Journal of Mathematical Analysis and Applications, 345:147-164, 2008.

[34] H. De Bie. Clifford algebras, Fourier transforms and quantum mechanics. Mathematical Methods in the Applied Sciences, To appear. 29 pages.

[35] H. De Bie and N. De Schepper. The fractional Clifford-Fourier transform. Complex Analysis and Operator Theory, To appear. 17 pages.

[36] H. De Bie, N. De Schepper, and F. Sommen. The class of Clifford-Fourier transforms. Journal of Fourier Analysis and Applications, 17:1198-1231, 2011.

[37] H. De Bie, B. Ørsted, P. Somberg, and V. Souček. The Clifford deformation of the Hermite semigroup. Preprint arXiv:1101.5551, Jan. 2011. Available at: http://adsabs . harvard.edu/abs/2011arXiv1101.5551D.

[38] H. De Bie, B. Ørsted, P. Somberg, and V. Souček. Dunkl operators and a family of realizations of osp(1|2). Transactions of the American Mathematical Society, 364:3875-3902, 2012. 
[39] H. De Bie and Y. Xu. On the Clifford-Fourier transform. International Mathematics Research Notices, 2011(22):5123-5163, 2011.

[40] N. De Schepper. Multidimensional Continuous Wavelet Transforms and Generalized Fourier Transforms in Clifford Analysis. PhD thesis, Ghent University, Belgium, 2006.

[41] M. A. Delsuc. Spectral representation of 2D NMR spectra by hypercomplex numbers. Journal of magnetic resonance, 77(1):119-124, Mar. 1988.

[42] C. F. Dunkl. Differential-difference operators associated to reflection groups. Transactions of the American Mathematical Society, 311:167-183, 1989.

[43] J. Ebling and G. Scheuermann. Clifford Fourier transform on vector fields. IEEE Transactions on Visualization and Computer Graphics, 11(4):469-479, JulyAug. 2005.

[44] J. Ebling and J. Scheuermann. Clifford convolution and pattern matching on vector fields. In Proceedings IEEE Visualization, volume 3, pages 193-200, Los Alamitos, CA, 2003. IEEE Computer Society.

[45] T. A. Ell. Hypercomplex Spectral Transformations. PhD thesis, University of Minnesota, June 1992.

[46] T. A. Ell. Quaternion-Fourier transforms for analysis of 2-dimensional linear time-invariant partial-differential systems. In Proceedings of the 32nd Conference on Decision and Control, pages 1830-1841, San Antonio, Texas, USA, 15-17 December 1993. IEEE Control Systems Society.

[47] T. A. Ell and S. J. Sangwine. Decomposition of 2D hypercomplex Fourier transforms into pairs of complex Fourier transforms. In M. Gabbouj and P. Kuosmanen, editors, Proceedings of EUSIPCO 2000, Tenth European Signal Processing Conference, volume II, pages 1061-1064, Tampere, Finland, 5-8 Sept. 2000. European Association for Signal Processing.

[48] T. A. Ell and S. J. Sangwine. Hypercomplex Fourier transforms of color images. IEEE Transactions on Image Processing, 16(1):22-35, Jan. 2007.

[49] R. R. Ernst, G. Bodenhausen, and A. Wokaun. Principles of Nuclear Magnetic Resonance in One and Two Dimensions. International Series of Monographs on Chemistry. Oxford University Press, 1987.

[50] M. Felsberg. Low-Level Image Processing with the Structure Multivector. PhD thesis, Christian-Albrechts-Universität, Institut für Informatik und Praktische Mathematik, Kiel, 2002.

[51] W. R. Hamilton. On a new species of imaginary quantities connected with a theory of quaternions. Proceedings of the Royal Irish Academy, 2:424-434, 1843. Online: http://www.maths.tcd.ie/pub/HistMath/People/ Hamilton/Quatern1/Quatern1.html.

[52] E. Hitzer. Multivector differential calculus. Advances in Applied Clifford Algebras, 12(2):135-182, 2002.

[53] E. Hitzer. Vector differential calculus. Memoirs of the Faculty of Engineering, Fukui University, 50(1):109-125, 2002.

[54] E. Hitzer. Quaternion Fourier transform on quaternion fields and generalizations. Advances in Applied Clifford Algebras, 17(3):497-517, May 2007.

[55] E. Hitzer. Tutorial on Fourier transformations and wavelet transformations in Clifford geometric algebra. In K. Tachibana, editor, Lecture notes of the 
International Workshop for 'Computational Science with Geometric Algebra' (FCSGA2007), pages 65-87, Nagoya University, Japan, 14-21 February 2007.

[56] E. Hitzer. Clifford (geometric) algebra wavelet transform. In V. Skala and D. Hildenbrand, editors, Proceedings of GraVisMa 2009, pages 94-101, Plzen, Czech Republic, 2-4 September 2009. Online: http://gravisma.zcu.cz/ GraVisMa-2009/Papers_2009/!_2009_GraVisMa_proceedings-FINAL .pdf.

[57] E. Hitzer. Real Clifford algebra $\operatorname{cl}(n, 0), n=2,3(\bmod 4)$ wavelet transform. In Simos et al. [88], pages 781-784.

[58] E. Hitzer. Directional uncertainty principle for quaternion Fourier transforms. Advances in Applied Clifford Algebras, 20(2):271-284, 2010.

[59] E. Hitzer. OPS-QFTs: A new type of quaternion Fourier transforms based on the orthogonal planes split with one or two general pure quaternions. In T. E. Simos, G. Psihoyios, C. Tsitouras, and Z. Anastassi, editors, NUMERICAL ANALYSIS AND APPLIED MATHEMATICS ICNAAM 2011: International Conference on Numerical Analysis and Applied Mathematics, volume 1389 of AIP Conference Proceedings, pages 280-283, Halkidiki, Greece, 19-25 September 2011.

[60] E. Hitzer. The Clifford Fourier transform in real Clifford algebras. In K. Guerlebeck, T. Lahmer, and F. Werner, editors, Proceedings 19th International Conference on the Application of Computer Science and Mathematics in Architecture and Civil Engineering, Weimar, Germany, 4-6 July 2012.

[61] E. Hitzer. Two-sided Clifford Fourier transform with two square roots of -1 in $C \ell_{p, q}$. In Proceedings of the 5th Conference on Applied Geometric Algebras in Computer Science and Engineering (AGACSE 2012), La Rochelle, France, 2-4 July 2012.

[62] E. Hitzer and R. Abłamowicz. Geometric roots of -1 in Clifford algebras $C \ell_{p, q}$ with $p+q \leq 4$. Advances in Applied Clifford Algebras, 21(1):121-144, 2010. Published online 13 July 2010.

[63] E. Hitzer and B. Mawardi. Uncertainty principle for the Clifford geometric algebra $c l(3,0)$ based on Clifford Fourier transform. In T. E. Simos, G. Sihoyios, and C. Tsitouras, editors, International Conference on Numerical Analysis and Applied Mathematics 2005, pages 922-925, Weinheim, 2005. Wiley-VCH.

[64] E. Hitzer and B. Mawardi. Uncertainty principle for Clifford geometric algebras $C \ell_{n, 0}, n=3(\bmod 4)$ based on Clifford Fourier transform. In Qian et al. [83], pages $47-56$.

[65] E. Hitzer, T. Nitta, and Y. Kuroe. Applications of Clifford's geometric algebra. Advances in Applied Clifford Algebras, 2012. accepted.

[66] E. M. S. Hitzer and B. Mawardi. Clifford Fourier transform on multivector fields and uncertainty principles for dimensions $n=2(\bmod 4)$ and $n=3$ ( mod 4). Advances in Applied Clifford Algebras, 18(3-4):715-736, 2008.

[67] G. Kaiser. Communications via holomorphic Green functions. In Clifford Analysis and its Applications, Kluwer NATO Science Series, 2001.

[68] G. Kaiser. Complex-distance potential theory, wave equations, and physical wavelets. Mathematical Methods in the Applied Sciences, 25:1577-1588, 2002. Invited paper, Special Issue on Clifford Analysis in Applications.

[69] G. Kaiser. Huygens' principle in classical electrodynamics: a distributional approach. Preprint arXiv:0906.4167, June 2009. 
[70] G. Kaiser, E. Heyman, and V. Lomakin. Physical source realization of complexsource pulsed beams. Journal of the Acoustical Society of America, 107:18801891, 2000.

[71] T. Kobayashi and G. Mano. Integral formulas for the minimal representation of $o(p, 2)$. Acta Applicandae Mathematicae, 86:103-113, 2005.

[72] X. Li. On the inverse problem for the Dirac operator. Inverse Problems, 23:919932, 2007.

[73] B. Mawardi, S. Adji, and J. Zhao. Clifford algebra-valued wavelet transform on multivector fields. Advances in Applied Clifford Algebras, 21(1):13-30, 2011.

[74] B. Mawardi and E. Hitzer. Clifford algebra $c l(3,0)$-valued wavelets and uncertainty inequality for Clifford Gabor wavelet transformation. Preprints of Meeting of the Japan Society for Industrial and Applied Mathematics, 16-18 September 2006.

[75] B. Mawardi and E. Hitzer. Clifford algebra $c l(3,0)$-valued wavelet transformation, Clifford wavelet uncertainty inequality and Clifford Gabor wavelets. International Journal of Wavelets, Multiresolution and Information Processing, 5(6):997-1019, 2007.

[76] B. Mawardi, E. Hitzer, and S. Adji. Two-dimensional Clifford windowed Fourier transform. In E. Bayro-Corrochano and G. Scheuermann, editors, $A p$ plied Geometric Algebras in Computer Science and Engineering, pages 93-106. Springer, London, 2010.

[77] B. Mawardi, E. Hitzer, A. Hayashi, and R. Ashino. An uncertainty principle for quaternion Fourier transform. Computers and Mathematics with Applications, 56(9):2411-2417, 2008

[78] B. Mawardi and E. M. S. Hitzer. Clifford Fourier transformation and uncertainty principle for the Clifford algebra $C \ell_{3,0}$. Advances in Applied Clifford Algebras, 16(1):41-61, 2006.

[79] M. Mitrea. Clifford Wavelets, Singular Integrals, and Hardy Spaces, volume 1575 of Lecture notes in mathematics. Springer, Berlin, 1994.

[80] M. Moshinsky and C. Quesne. Linear canonical transformations and their unitary representations. Journal of Mathematical Physics, 12:1772-1780, 1971.

[81] V. Namias. The fractional order Fourier transform and its application to quantum mechanics. IMA Journal of Applied Mathematics, 25(3):241-265, 1980.

[82] H. Ozaktas, Z. Zalevsky, and M. Kutay. The Fractional Fourier Transform. Wiley, Chichester, 2001.

[83] T. Qian, M. I. Vai, and Y. Xu, editors. Wavelet Analysis and Applications, Applied and Numerical Harmonic Analysis. Birkhäuser Basel, 2007.

[84] S. J. Sangwine. Fourier transforms of colour images using quaternion, or hypercomplex, numbers. Electronics Letters, 32(21):1979-1980, 10 Oct. 1996.

[85] S. J. Sangwine. Biquaternion (complexified quaternion) roots of -1. Advances in Applied Clifford Algebras, 16(1):63-68, June 2006.

[86] S. J. Sangwine and T. A. Ell. The discrete Fourier transform of a colour image. In J. M. Blackledge and M. J. Turner, editors, Image Processing II Mathematical Methods, Algorithms and Applications, pages 430-441, Chichester, 2000. Horwood Publishing for Institute of Mathematics and its Applications. Proceedings Second IMA Conference on Image Processing, De Montfort University, Leicester, UK, September 1998. 
[87] S. J. Sangwine and N. Le Bihan. Quaternion polar representation with a complex modulus and complex argument inspired by the Cayley-Dickson form. Advances in Applied Clifford Algebras, 20(1):111-120, Mar. 2010. Published online 22 August 2008.

[88] T. E. Simos, G. Psihoyios, and C. Tsitouras, editors. NUMERICAL ANALYSIS AND APPLIED MATHEMATICS: International Conference on Numerical Analysis and Applied Mathematics 2009, volume 1168 of AIP Conference Proceedings, Rethymno, Crete (Greece), 18?-22 September 2009.

[89] F. Sommen. A product and an exponential function in hypercomplex function theory. Applicable Analysis, 12:13-26, 1981.

[90] F. Sommen. Hypercomplex Fourier and Laplace transforms I. Illinois Journal of Mathematics, 26(2):332-352, 1982.

[91] F. Sommen. Hypercomplex Fourier and Laplace transforms II. Complex Variables, 1(2-3):209-238, 1983.

[92] L. Traversoni. Quaternion wavelet problems. In Proceedings of 8th International Symposium on Approximation Theory, Texas A\& M University, Jan. 1995.

[93] J. Zhao. Clifford algebra-valued admissible wavelets associated with admissible group. Acta Scientarium Naturalium Universitatis Pekinensis, 41(5):667-670, 2005.

[94] J. Zhao and L. Peng. Quaternion-valued admissible wavelets associated with the 2D Euclidean group with dilations. Journal of Natural Geometry, 20(1/2):21-32, 2001.

Fred Brackx

Department of Mathematical Analysis, Faculty of Engineering

Ghent University, Galglaan 2, B-9000 Gent, Belgium.

e-mail: Freddy.Brackx@ugent. be

Eckhard Hitzer

College of Liberal Arts, Department of Material Science,

International Christian University,

181-8585 Tokyo, Japan

e-mail: hitzer@icu.ac.jp

Stephen J. Sangwine

School of Computer Science and Electronic Engineering,

University of Essex, Wivenhoe Park, Colchester, CO4 3SQ, UK.

e-mail: sjs@essex.ac.uk 